\title{
Metastatic Renal Cell Carcinoma to the Nasal Cavity, but after 17 Years
}

\author{
Allon $\mathrm{I}^{1,2}$, Sinelnikov $\mathrm{I}^{1}$, Schaffer $\mathrm{M}^{2,3}$, Forer $\mathrm{B}^{4}$ and Livoff $\mathrm{A}^{1}$ \\ ${ }^{1}$ Institute of pathology, Barzilai University Medical Center, Ashkelon, Israel \\ ${ }^{2}$ Faculty of Health sciences, Ben-Gurion University, Beer-Sheba, Israel \\ ${ }^{3}$ Institute of Oncology, Barzilai University Medical Center, Ashkelon, Israel \\ ${ }^{4}$ Department of Otolaryngology, Barzilai University Medical Center, Ashkelon, Israel
}

\begin{abstract}
We present a case of a 64-year-old man with a bilateral nasal obstruction. An endoscopic biopsy taken from the mass revealed a vascular clear cell tumor underneath an intact Schneiderian membrane compatible with a renal cell carcinoma. Following inquiry, it was revealed that the patient had gone through right radical nephrectomy for renal cell carcinomas 17 years prior to admission, a fact that was not known when the biopsy was taken. A following workup showed areas suspicious for metastases in lungs and maxillary sinuses. The patient started treatment with Sunitinib. On five months follow- up, the lung lesions had regressed.
\end{abstract}

Keywords: Nasal cavity • Metastatic renal cell carcinoma • Histopathology • PAX 8

\section{Introduction}

Patients with renal cell carcinoma (RCC) develop metastases in approximately $30 \%$ of cases [1]. Common sites of metastases of RCC are lungs, liver, bone, brain, and adrenal glands, but RCC can metastasize to any organ [1]. Metastases to the nasal cavity or paranasal sinuses are rare, and only several case reports have been published [2-8]. To our knowledge case series of metastatic RCC to the paranasal sinuses have not been published. Distant metastasis of RCC may be found at presentation or can develop years after initial diagnosis [1]. The prognosis of patients with metastatic RCC is poor. In this paper, we report the case of a 64 year old patient who developed metastasis to the left paranasal sinuses 17 years after nephrectomy for RCC.

\section{Case Report}

A 64-year-old man was referred to the otolaryngology outpatient clinic due to a bilateral nasal obstruction lasting for the last few months, more on the left and occasional minor bleeding from the left nostril. The patient denied rhinorrhea, anosmia or pain.

Computed tomography (CT) of the paranasal sinuses showed a polypoid mass protruding from the ethmoids into the nasal cavity (Figure 1). No evidence of expansion of invasion of skull base or orbit was noted.

Endoscopic examination of the left nasal cavity revealed a polypoid mass bulging from the ethmoid into the nasal cavity. The surface area of the mass was smooth with no evidence of ulceration or necrosis. The right nasal cavity was without evidence of disease.

The differential diagnosis included an inflammatory process (chronic sinusitis, fungal non-invasive sinusitis) and neoplastic disease (benign and rarely, malignant tumors). An endoscopic biopsy was taken and the tissue was submitted to pathological evaluation.

*Address for Correspondence: Dr. Irit Allon, Unit of Pathology, Barzilai Medical Center, Hahistadrut st. 2, Ashkelon, Israel, E-mail: allonirit1@gmail.com

Copyright: (c) 2020 Allon I, et al. This is an open-access article distributed under the terms of the Creative Commons Attribution License, which permits unrestricted use, distribution, and reproduction in any medium, provided the original author and source are credited.
On pathologic examination a tumor was present under the nasal lining epithelium and the respiratory pseudostratified columnar epithelium was undisturbed (Figures 2A-2C). The tumor was vascular with numerous blood sinusoids. The tumor cell population was composed of both cells with optically clear cytoplasm and cells that contained eosinophilic granular cytoplasm (Figures 2A-2C). There was no pronounced cellular atypia and no marked mitotic or apoptotic bodies. The impression strongly favored metastatic RCC. Differential diagnoses included other primaries of metastatic clear cell carcinoma (e.g. liver, thyroid), clear cell sarcoma, malignant melanoma and clear cell oncocytoma. An immunohistochemical panel was used in order to verify the diagnosis. The tumor cells were positive for pankeratin (AE1/3, CAM5.2), CD10, RCC Ag, PAX8, vimentin and Ki-67 labeling = 15\% (Figures $3 A-3 F)$.

A revision medical history inquiry revealed a history of radical nephrectomy due to RCC 17 years prior to admission. Oncologic follow-up for a few years after initial surgery was without evidence of recurrent disease. Thus, a pathological diagnosis of metastatic RCC of clear cell type (grade 1) was rendered.

A Staging workup with fluorine-18, flouro-D- glucose Positron emission tomography scan (FDG- PET-CT) was done showing numerous multi-sized nodes in right and left lower lobes of lungs, measuring up to 34 millimeters

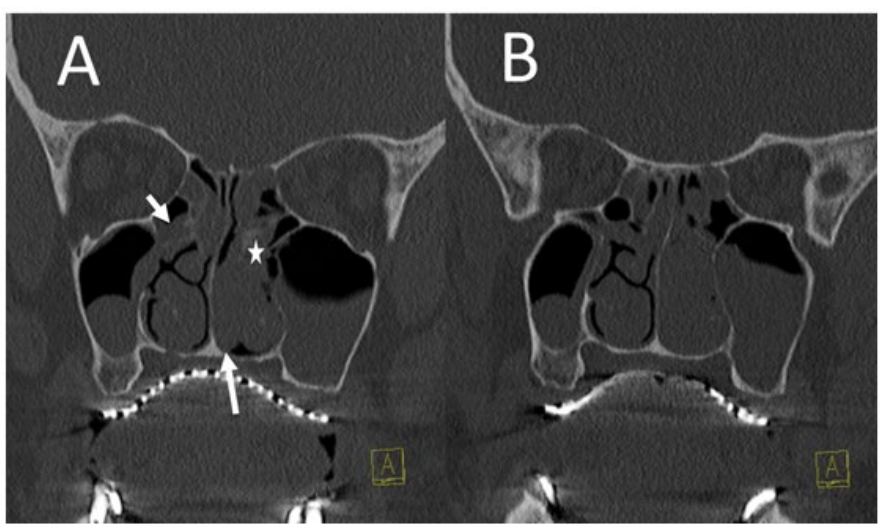

Figure 1. Coronal CT imaging of the paranasal sinuses. A section at the level of the anterior $(A)$ and posterior (B) ethmoids. A polypoid mass can be seen originating from the ethmoid complex (star) and expanding downwards to the nasal floor. The lower border of the mass appears smooth (long arrow). No evidence of expansion and orbital or skull base invasion is seen. Ethmoid and maxillary opacifications can also be seen on the right paranasal sinuses (short arrow). 


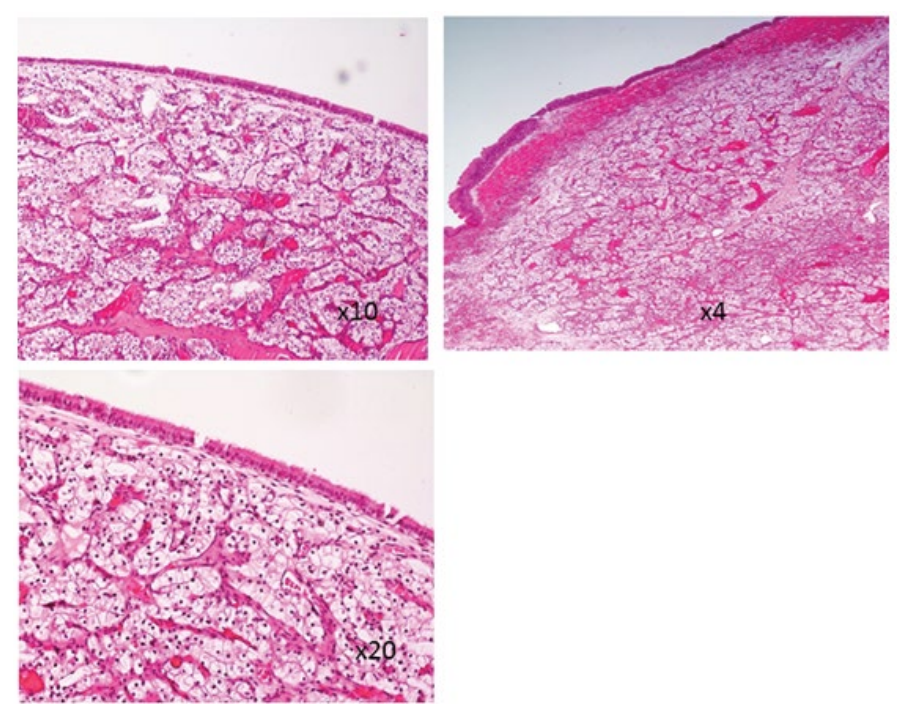

Figure 2. A-C: HEE stain showing a clear cell tumor immediately underneath the shneiderian membrane. The clear cells are arranged in nests separated by a prominent sinusoidal vascular network. The cells have well defined borders. (4x, 10x, 20x).
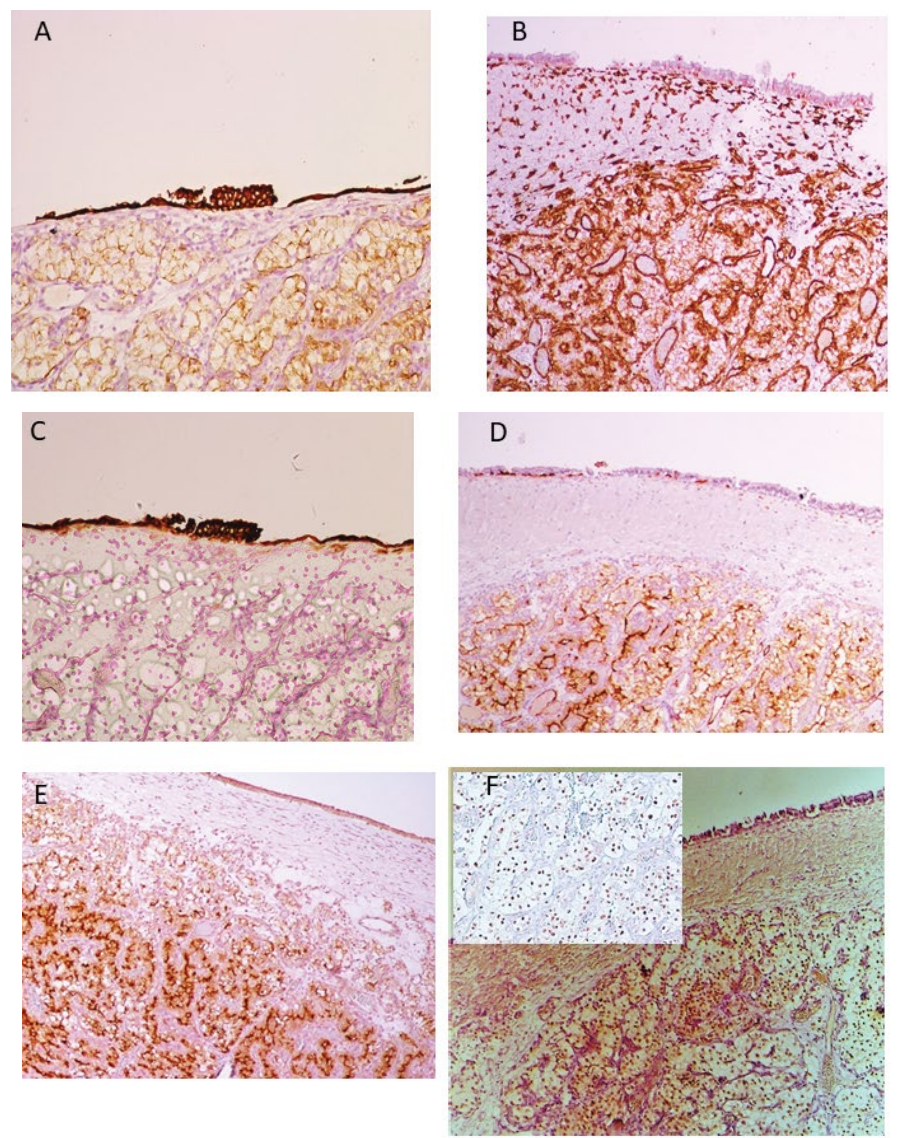

Figure 3. Immunohistochemically, the tumor cells were positive to: $a-A E 1 / 3, b-V i m e n t i n$, c-CK7, d-CD10, e-RCCAg, f-PAX8 (20x).

mildy enhanced but suspicious for metastatic disease. In addition, fullness of ethmoid and maxillary sinuses was noted, with no enhancement or pathological intake. The multi-disciplinary tumor board team decided that a metastatic disease could not be ruled out on the basis of multiple foci, and their size. The patient started a daily systemic treatment with P.O. as $50 \mathrm{mg}$ Sutent (Sunitinib), a multi targeted receptor tyrosine kinase inhibitor. On five months follow up CT scan, paranasal sinuses were without a significant change but the lesions in the lungs were reduced by $4 \mathrm{~mm}$ each.

\section{Discussion}

About $30 \%$ of patients with RCC develop metastases after nephrectomy, which occur within 5 years [1]. In the present case, the interval of nephrectomy for RCC and emergence of nasal metastasis was as long as 17 years. This long interval has been reported in the past [8]. In the present study, the RCC is clear cell RCC and Fuhrman's nuclear grade was grade I [9]. This type of RCC shows lower possibility of metastasis; however, the present patient showed nasal metastasis 17 years after the nephrectomy [10]. These findings indicate that long-term follow-up is needed in patients with RCC and the unique pre metastatic niche involved in RCC.

The tumor presented characteristic morphological features that raised the possibility of metastatic RCC which are prominent vascular structures and epithelialclearcellcomponentwithoutprominentatypia. Immunohistochemically, RCC has a characteristic signature, which encompasses keratin and vimentin positivity, CD10, RCC Ag and PAX 8 positivity.

Treatment of metastatic RCC includes a multi targeted receptor tyrosine kinase inhibitor such as sunitinib $50 \mathrm{mg} / \mathrm{day}$. Other possibilities are Sorafanib, Pazopanib, Temsirolimus [11].

The prognosis of patients with metastatic RCC is poor with survival ranging between 10.2 to 22 months [12,13]. In the present case five months follow up showed shrinkage of the metastatic lesions in the lung although the systemic sequel included brain infarction, right hydronephrosis, and myelodysplastic syndrome. Biological treatments usually show gradual results and it takes several months to detect the improvement.

\section{Conclusion}

In conclusion, we reported a case of metastatic RCC of the nasal cavity. The pathological diagnosis was made without knowledge of the status of the previous malignancy. The time interval between nephrectomy and nasal cavity metastasis was as long as 17years. The pre-metastatic niche of RCC is unique as it enables a relatively late stage formation of the metastatic deposit.

\section{Financial Disclosure}

No funding was received for this study.

\section{Conflict of Interest}

The authors declare no conflict of interest.

\section{References}

1. Flanigan, Robert C, Steven C Campbell, Joseph I Clark and Maria M Picken. "Metastatic Renal Cell Carcinoma". Curr Treat Options Oncol 4 (2003): 385-390.

2. Hayes, Elizabeth, Alfred L Weber, Kenneth R Davis and Jr FG Arrigg. "Metastatic Renal Cell Carcinoma Manifesting as a Nasal Mass: CT Findings". J Comput Assist Tomogr 9 (1985): 387-389.

3. Vreugde S, R Duttmann, A Halama and P Deron. "Metastasis of Renal Cell Carcinoma to the Nose and Paranasal Sinus". Acta Otorynolaryngol Belg 53 (1999): 129-131.

4. Yee, Lynne Lim Hsueh, and Christopher Goh Hood Keng. "A Rare Case of Renal Cell Carcinoma Metastatic to the Sinonasal Area". Ear Nose Throat J 80 (2001): 462-467.

5. Nason, Robert and Ricardo L Carrau. "Metastatic Renal Cell Carcinoma to the Nasal Cavity". Am J Otolaryngol 25 (2004): 54-57.

6. Dinçbas, FO, B Atalar, DC Oksuz and FV Aker, et al. "Unusual Metastasis of Renal Cell Carcinoma to the Nasal Cavity". J BUON 9 (2004): 201-204. 
7. Lee, HM, HJ Kang and SH Lee. "Metastatic Renal Cell Carcinoma Presenting Epistaxis". Eur Arch Otorhinolaryngol 262 (2005): 69-71.

8. Ziari M, Shen S, Amaro RJ, Teh BS. "Metastatic Renal Cell Carcinoma to the Nose and Ethmoid Sinus". Urology 67 (2006): 199.

9. Klatte, Tobias, David B Seligson, Jeffrey LaRochelle and Brian Shuch, et al. "Molecular Signatures of Localized Clear Renal Cell Carcinoma to Predict DiseaseFree Survival after Nephrectomy". Cancer Epidermol Biomarkers Prev 18 (2009): 894-900.

10. Amin, Mahul B, Mitual B Amin, Pheroze Tamboli and Javid Javidan, et al.
"Prognostic Impact of Histologic Subtyping of Adult Renal Epithelial Neoplasms: An Experience Of 405 Cases". Am J Surg Pathol 26 (2002): 281-291.

11. www.ncen.org/professionals/physician_gls/pdf/kidney.pdf

12. Motzer RJ, Bacik J, Schwarz LH, Reuter V, Russo P, Marison S, Mazumdar M. "Prognostic Factors for Survival in Previously Treated Patients with Metastatic Renal Cell Carcinoma”. J Clin Oncol 22 (2004): 453-463.

13. Motzer, Robert J, Jennifer Bacik, Lawrence H Schwartz and Victor Reuter, et al. "Prognostic Factors for Overall Survival in Patients with Metastatic Renal Cell Carcinoma Treated with Vascular Endothelial Growth Factor-Targeted Agents: Results from a Large, Multicenter Study". J Clin Oncol 27 (2009): 569-579.

How to cite this article: Allon Irit, Igor Sinelnikov, Moshe Schaffer and Boaz Forer, et al. "Metastatic Renal Cell Carcinoma to the Nasal Cavity, but after 17 Years." J Mol Genet Med 14 (2020): 454 doi: 10.37421/jmgm.2020.14.454 